\title{
Dcpo models of $T_{1}$ spaces
}

\author{
Zhao Dongsheng and Xi Xiaoyong \\ 1 Mathematics and Mathematics Education \\ National Institute of Education Singapore \\ Nanyang Technological University \\ 1 Nanyang Walk \\ Singapore 637616 \\ dongsheng.zhao@nie.edu.sg \\ 2 Department of Mathematics \\ Jiangsu Normal University \\ Jiangsu China \\ littlebrook@jsnu.edu.cn
}

A poset model of a topological space $X$ is a poset $P$ together with a homeomorphism $\phi: X \longrightarrow \operatorname{Max}(P)(\operatorname{Max}(P)$ is the subspace of the Scott space $\Sigma P$ consisting of maximal points of $P$ ). In [11] (also in 2]), it was proved that every $T_{1}$ space has a bounded complete algebraic poset model. It is, however still unclear whether each $T_{1}$ space has a dcpo model. In this paper we give a positive answer to this problem. In section 1 , we show that every $T_{1}$ space has a dcpo model. In section 2 , we prove that a $T_{1}$ space is sober if and only if its dcpo model constructed in section 1 is a sober dcpo. These results provide us with a method to construct non-sober dcpos from any non-sober $T_{1}$ spaces. In section 3 , for some special spaces we construct a more concrete dcpo model.

\section{Dcpo models of $T_{1}$ spaces}

Theorem 1. [11] Every $T_{1}$ space has a bounded complete algebraic poset model.

Remark 1. Let $X$ be a $T_{0}$ space and $\mathcal{A}$ be the set of all filters of open sets of $X$ that has a nonempty intersection. Then $(\mathcal{A}, \subseteq)$ is a bounded complete algebraic poset and the following properties hold:

(1) the mapping $\phi: X \longrightarrow \Sigma \mathcal{A}$, defined by $\phi(x)=N(x), x \in X(N(x)$ is the filter of open neighbourhood of $x$ ), is a topological embedding;

(2) $\operatorname{Max}(\mathcal{A}) \subseteq \phi(X)$, and $X$ is $T_{1}$ if and only if $\phi(X)=\operatorname{Max}(\mathcal{A})$;

(3) every member of $\mathcal{A}$ is below some $N(x)$, so the closure of $\phi(X)$ in $\Sigma \mathcal{A}$ equals $\mathcal{A}$.

Thus every $T_{0}$ space is homeomorphic to a dense subspace of the Scott space of a bounded complete algebraic poset.

A poset $\mathrm{P}$ is called a local dcpo (or bounded complete dcpo) if every upper bounded directed subset has a supremum [12]. Clearly, every bounded complete poset is a local dcpo.

Lemma 1. For any local dcpo $A$, there is a dcpo $\hat{A}$ such that $\operatorname{Max}(A)$ and $\operatorname{Max}(\hat{A})$ are homeomorphic.

A poset $P$ is locally quasicontinuous if for each $a \in P$, the sub poset $\downarrow a$ is quasicontinuous.

Lemma 2. If $A$ is a bounded complete algebraic poset, then the dcpo $\hat{A}$ constructed in Lemma 1 from $A$ is locally quasicontinuous. 
Given a $T_{1}$ space, by Theorem 1 there is a bounded complete algebraic poset $A$ such that $\operatorname{Max}(A)$ is homeomorphic to $X$. Since every bounded complete poset is a local dcpo, by Lemma 1 there is a dcpo $\hat{A}$ such that $\operatorname{Max}(A)$ is homeomorphic to $\operatorname{Max}(A)$. All these deduce the first main result of this paper.

Theorem 2. Every $T_{1}$ topological space has a dcpo model.

Remark 2. By Lemma 2, we can actually deduce that every $T_{1}$ space has a dcpo model that is locally quasicontinuous.

Proposition 1. Every $T_{0}$ space can be embedded, as a dense subset, into to the Scott space of an algebraic dcpo.

\section{Dcpo models of sober spaces}

Proposition 2. If $P$ is a poset such that $\Sigma P$ is sober, then the subspace $\operatorname{Max}(P)$ of $\Sigma P$ is sober.

By Proposition III-3.7 of [3], the Scott space of every quasicontinuous dcpo is sober, so we have the following result.

Corollary 1. For any quasicontinuous dcpo, in particular for any continuous dcpo $P, \operatorname{Max}(P)$ is sober.

Lemma 3. Let $A$ be a bounded complete algebraic poset and $\hat{A}$ be the dcpo constructed from $A$ in Lemma 1. If $\operatorname{Max}(\hat{A})$ is sober then $\Sigma \hat{A}$ is sober.

From the above two results we deduce the following.

Theorem 3. A topological space $X$ has a dcpo model whose Scott topology is sober if and only if $X$ is $T_{1}$ and sober.

We call a dcpo $P$ sober, if its Scott topology is sober. Johnstone first constructed a nonsober dcpo in [5], then Isbel gave a non-sober complete lattice [4. Finding a non-sober dcpo is surprisingly uneasy (as far as the authors know, up-to-date, only three such dcpos have been constructed).

Now if $X$ is a $T_{1}$ and non-sober space, then the dcpo model constructed for $X$ in Theorem 2 is non-sober.

For a specific example, let $Y$ be an infinite set and $\tau$ be the co-finite topology on $Y$ (i.e. $U \in \tau$ if and only if either $U=\emptyset$ or $Y-U$ is a finite set). Then $(Y, \tau)$ is $T_{1}$ and non-sober.

Proposition 3. Let $Q$ be a dcpo model of $(Y, \tau)$. Then $Q$ is a non-sober dcpo.

\section{Dcpo models of some special spaces}

Let $\omega_{1}$ be the first non-countable ordinal and $W=\left[0, \omega_{1}\right)$ be the set of all ordinals less than $\omega_{1}$. Thus $\mathrm{W}$ consists of all finite and infinite countable ordinals.

Remark 3. The following facts are well known. 1) $|W|=\aleph_{1}$.

2) For any countable subset $D \subseteq W, \sup D \in W$, here the $\sup D$ is taken with respect to the usual linear order on ordinals.

3) For any $\alpha \in W,\{\beta: \beta \leq \alpha\}$ is a finite or countably infinite subset of $W$. 
Let $\tau$ be the co-countable topology on $W$, that is $U \in \tau$ if and only if either $U=\emptyset$ or $W-U$ is a finite or countably infinite set. We now construct a simpler dcpo model for $(W, \tau)$.

Let $P_{\aleph_{0}}=\left\{x_{\alpha}: x \in W, \alpha \in W\right\} \cup W$. The order on $P$ is defined as follows:

(i) $x_{\alpha} \leq y_{\beta}$ iff $\alpha=\beta$ and $x \leq y$;

(ii) $x_{\alpha}<\alpha$;

(iii) $x_{\alpha}<\beta$, where $\alpha \neq \beta$, iff $x<\beta$.

Then $P_{\aleph_{0}}$ is a dcpo and $\operatorname{Max}\left(P_{\aleph_{0}}\right)=W$.

Lemma 4. (1) For any finite or countably infinite subset $A \subseteq W$, there is a Scott closed set $F$ of $P_{\aleph_{0}}$ such that $A=F \cap W$.

(2) For any Scott closed set $F$ of $P_{\aleph_{0}}$, either $W \subseteq F$ or $W-F$ is at most a countably infinite set.

Proposition 4. The dcpo $P_{\aleph_{0}}$ defined above is a model of the space of set $W=\left[0, \omega_{1}\right)$ with the co-countable topology.

As $W$ is not sober, its dcpo model $P_{\aleph_{0}}$ is non-sober in the Scott topology. This gives another example of non-sober dcpo.

In general, let $\aleph$ be a cardinal and $W_{\aleph}$ be the set of all ordinals $\alpha$ with $|\alpha|<\aleph$. The $\aleph$-complementary topology $\mu$ on $W_{\aleph}$ is the topology whose open sets are either $\emptyset$ or whose complement has cardinal less than or equal to $\aleph$. Then we can construct a dcpo model of $\left(W_{\aleph}, \tau\right)$ in a similar way as for $(W, \tau)$.

Remark 4. (1) Following the method as for Lemma 4 let $\mathbb{N}$ be the set of all natural numbers and $\tau$ the co-finite topology on $\mathbb{N}$. Let $P=\left\{n_{k}: n, k \in \mathbb{N}\right\} \cup \mathbb{N}$. Define the partial order $\leq$ on $P$ by

$$
m_{k} \leq n \text { for any } k \leq n, n_{k} \leq m_{l} \text { iff } m=n \text { and } k \leq l .
$$

Then $P$ is a dcpo model of $(\mathbb{N}, \tau)$ where $\tau$ is the co-finite topology.

(2) In [5, Johnstone gives an example of a dcpo whose Scott topology is not sober (this is the first such example ever constructed). One can verify that this dcpo isomorphic to the dcpo $P$ defined in (1).

A dcpo model $P$ of a $T_{1}$ space $X$ is said to satisfy the Lawson condition if $X$ is homeomorphic to $\operatorname{Max}(P)$ with the inherited Lawson topology on $P$. Lawson proved that a space has a continuous dcpo model satisfying Lawson condition that has a countable base iff the space is Polish [7. In [11, it was proved that a space has an algebraic poset model satisfying Lawson condition iff it is zero-dimensional.

Theorem 4. If a space is zero dimensional then it has a dcpo model satisfying Lawson condition.

\section{References}

[1] Birhoff, G. Lattice Theory, 3rd ed., American mathematical Society, Providence, Rhode Island, 1973.

[2] Erné, M.: Algebraic models for T1-spaces, Topology and its Applications 158(2011), 7:945-962.

[3] Gierz, G., Hofmann, K. H., Keimel, K., Lawson, J. D., Mislove, M. W. and Scott, D. S.: Continuous lattices and Domains, Encyclopedia of Mathematics and Its Applications, Vol.93, Cambridge University Press, 2003. 
[4] Isbell, J. R.:Completion of a construction of Johnstone, Proc. Amer. Math. Soc., 85(1982), 333334.

[5] Johnstone, P.: Scott is not always sober, In: Continuous Lattices, Lecture Notes in Math. 871, Springer-Verlag, (1981), 282-283.

[6] Johnstone, P.: Stone spaces, Cambridge University Press, 1982.

[7] Lawson, J. D.: Spaces of maximal points, Math. Structures comput. Sci. 7(1997), 5: 543-555.

[8] Liang, L. and Klause, K.: Order environment of topological spaces, Acta Math. Sinica 20(2004), 5: $943-948$.

[9] Martin, K.: Ideal models of spaces, Theoretical computer science 305(2003), 277-297.

[10] Scott, D. S.: Continuous Lattices, In: Toposes, Algebraic Geometry and Logic, Lecture Notes in Math. 274, Springer-Verlag, (1972), 97-136.

[11] Zhao, D.: Poset models of topological spaces, In: Proceeding of International Conference on Quantitative Logic and Quantification of Software, Global - Link Publisher, (2009), 229-238.

[12] Zhao, D. and Fan, T.: dcpo-completion of posets, Theoretical Computer Science 411(2010), 21672173. 